\title{
Brain injury in the international multicenter randomized SafeBoosC phase II feasibility trial: cranial ultrasound and magnetic resonance imaging assessments
}

\author{
Anne M Plomgaard ${ }^{1}$, Cornelia Hagmann², Thomas Alderliesten ${ }^{3}$, Topun Austin ${ }^{4}$, Frank van Bel ${ }^{3}$, Olivier Claris ${ }^{5}$, \\ Eugene Dempsey ${ }^{6}$, Axel Franz ${ }^{7}$, Monica Fumagalli ${ }^{8}$, Christian Gluud ${ }^{9}$, Gorm Greisen ${ }^{1}$, Simon Hyttel-Sorensen ${ }^{1}$, Petra Lemmers ${ }^{3}$, \\ Adelina Pellicer ${ }^{10}$, Gerhard Pichler ${ }^{11}$ and Manon Benders ${ }^{3}$
}

BACKGROUND: Abnormal cerebral perfusion during the first
days of life in preterm infants is associated with higher grades of
intraventricular hemorrhages and lower developmental score.
In SafeBoosC II, we obtained a significant reduction of cerebral
hypoxia by monitoring cerebral oxygenation in combination
with a treatment guideline. Here, we describe (i) difference in
brain injury between groups, (ii) feasibility of serial cranial ultra-
sound (CUS) and magnetic resonance imaging (MRI), (iii) local
and central cUS assessment.

METHODS: Hundred and sixty-six extremely preterm infants were included. cUS was scheduled for day 1, 4, 7, 14, and 35 and at term-equivalent age (TEA). cUS was assessed locally (unblinded) and centrally (blinded). MRI at TEA was assessed centrally (blinded). Brain injury classification: no, mild/moderate, or severe.

RESULTS: Severe brain injury did not differ significantly between groups: cUS (experimental 10/80, control 18/77, $P=0.32)$ and $M R I(5 / 46$ vs. $3 / 38, P=0.72)$. Kappa values for local and central readers were moderate-to-good for severe and poor-to-moderate for mild/moderate injuries. At TEA, cUS and MRI were assessed in 72 and 64\%, respectively.

CONCLUSION: There was no difference in severe brain injury between groups. Acquiring CUS and MRI according the standard operating procedures must be improved for future trials. Whether monitoring cerebral oxygenation during the first $72 \mathrm{~h}$ of life prevents brain injury should be evaluated in larger multicenter trials.

$\mathbf{T}$ he circulatory adaption to birth is problematic for the vulnerable preterm infant. The immature respiratory and circulatory systems may cause episodes of cerebral hypoxia, which may lead to brain injury. Near-infrared spectroscopy (NIRS) has been used to monitor tissue oxygenation, and abnormal cerebral perfusion has been associated with higher grades of intraventricular hemorrhages and lower neurodevelopmental scores at $2 \mathrm{y}$ of age $(1,2)$. The multicenter feasibility trial SafeBoosC II (3) demonstrated that cerebral NIRS in combination with a dedicated treatment guideline (4) was able to reduce the median burden of cerebral hypo- and hyperoxia by $58 \%$ (95\% confidence interval: $35-73 \%$; $P<0.001$ ) compared with a control group with blinded collection of NIRS data and treatment as usual (5). Hypoxia was reduced, while hyperoxia was unaffected. Whether the experimental intervention prevents brain injury remains to be determined.

Cranial ultrasound (cUS) is the most widely used neuroimaging tool for assessing brain injury in preterm infants, and cUS findings are often reported as an outcome measure in randomized clinical trials. The advantages of cUS are that it causes minimal disturbance to the preterm infant, has no side effects, can be conducted at the bedside, is easy to repeat as often as indicated, and is relatively inexpensive. Furthermore, severe brain injury as assessed by cUS correlates well with later neurodevelopmental outcome; hence, cUS, especially sequentially, can be used to predict later neurodevelopmental outcome (6-9). Magnetic resonance imaging (MRI) at termequivalent age (TEA) though is superior to cUS in predicting neurodevelopmental outcome and cerebral palsy at $2 \mathrm{y}$ of age $(10,11)$. More recently, it has been suggested that MRI can be used for the evaluation of neuroprotective treatments by quantitative applications (12-14) as subtle differences in brain development can be detected (15). Thus, MRI may reduce the number of infants needed for future randomized neuroprotective trials. Additionally, MRI has been suggested for routine use in clinical settings for high-risk extremely preterm infants (13).

In SafeBoosC II, brain injury assessed by serial cUS was a secondary outcome and MRI at TEA was an explorative outcome. The aims of the present study were (i) to assess the difference in brain injury between the two treatment groups of

\footnotetext{
'Department of Neonatology, Rigshospitalet, Copenhagen University Hospital, Copenhagen, Denmark; ${ }^{2}$ Clinic of Neonatology, University of Zurich, Zurich, Switzerland; ${ }^{3}$ University Medical Center Utrecht, Wilhelmina Children's Hospital, Utrecht, The Netherlands; ${ }^{4}$ Rosie Maternity Hospital Cambridge University Hospitals NHS Foundation Trust, Cambridge, UK; ${ }^{5}$ Department of Neonatology, Hospital Femme Mere Enfants, Bron, France; ${ }^{6}$ Department of Paediatrics and Child Health, University College Cork, Cork, Ireland; ${ }^{7}$ Department of Neonatology, University of Tuebingen, Tübingen, Germany; ${ }^{8} \mathrm{NICU}$, Fondazione IRCCS Ca' Granda Ospedale Maggiore Policlinico, Milan, Italy; ${ }^{9} \mathrm{Copenhagen}$ Trial Unit, Centre for Clinical Intervention Research, Rigshospitalet, Copenhagen University Hospital, Copenhagen, Denmark; ${ }^{10}$ Department of Neonatology, La Paz University Hospital, Madrid, Spain; ${ }^{11}$ Department of Pediatrics, Medical University of Graz, Graz, Austria. Correspondence: Anne M Plomgaard (amplomgaard@gmail.com) Received 12 May 2015; accepted 31 August 2015; advance online publication 13 January 2016. doi:10.1038/pr.2015.239
} 
the SafeBoosC II trial, (ii) to describe the feasibility of neuroimaging (cUS and MRI) according to a standard operating procedure, and (iii) to explore the differences between interobserver agreement of central and local readings of serial cUS in this randomized multicenter feasibility trial.

\section{RESULTS}

Birth weight and gestational age did not differ between the experimental and the control groups (Table 1).

Table 1. Patient characteristics

\begin{tabular}{|c|c|c|}
\hline & $\begin{array}{l}\text { Experimental } \\
\text { group }\end{array}$ & Control group \\
\hline Infants included in SafeBoosC II & $n=86$ & $n=80$ \\
\hline $\begin{array}{l}\text { Gestational age (wk), median } \\
\text { (range) }\end{array}$ & $26.9(23.9-27.9)$ & $26.9(23.4-27.9)$ \\
\hline Birth weight (g), median (range) & $806(410-1,286)$ & $880(480-1,330)$ \\
\hline Infants with central overall cUS score & $n=80$ & $n=77$ \\
\hline $\begin{array}{l}\text { Gestational age (wk), median } \\
\text { (range) }\end{array}$ & $26.6(23.9-27.9)$ & $27.0(23.4-27.9)$ \\
\hline Birth weight (g), median (range) & $806(410-1,286)$ & $880(490-1330)$ \\
\hline Infants with MRI at any time & $n=49$ & $n=38$ \\
\hline $\begin{array}{l}\text { Gestational age (wk), median } \\
\text { (range) }\end{array}$ & $27.0(23.9-27.9)$ & $26.7(24.0-27.9)$ \\
\hline Birth weight (g), median (range) & $806(520-1,180)$ & $900(515-1,330)$ \\
\hline $\begin{array}{l}\text { Infants with MRI at corrected age } \\
40-44 \text { wk }\end{array}$ & $n=28$ & $n=29$ \\
\hline $\begin{array}{l}\text { Gestational age (wk), median } \\
\text { (range) }\end{array}$ & $26.7(23.9-27.9)$ & $27.0(24.9-27.9)$ \\
\hline Birth weight (g), median (range) & $800(520-1,180)$ & $934(700-1,330)$ \\
\hline
\end{tabular}

\section{Differences in Brain Injury Between Groups \\ Cranial ultrasound}

Local reading. The distribution of brain injury severity did not differ at any time-point between the experimental and the control groups; neither did the overall cUS-score.

Central reading. The raw and unadjusted $\mathrm{P}$ values for the between-group analysis showed a significant difference in the distributions of brain injury on day $1(P=0.043)$ and at TEA $(P=0.01)$, whereas the secondary outcome of the SafeBoosC II trial, the overall cUS score, did not differ significantly between the groups $(P=0.053)$. The number of severe injuries was lower in the experimental than in the control group, whereas the number of mild injuries was higher in the experimental than in the control group (Table 2). The odds ratio of severe brain injury vs. no severe brain injury was insignificant at all time points and for the overall cUS score. There were fewer severe intracranial hemorrhages (IVH grade III and periventricular hemorrhagic infractions) in the experimental group $(8 / 80(10 \%))$ than in the control group $(14 / 77(18.2 \%))$, but this was not significant (odds ratio 0.50 (95\% confidence interval: $0.20-1.27)$ ).

In 25 of the 28 preterm infants with severe brain injury, this was detected within the first $7 \mathrm{~d}$ of life. In the remaining three infants, severe brain injury on cUS was diagnosed by day 35 (posthemorrhagic ventricular dilatation, day 14; local cystic lesion, day 35; and cerebral atrophy, day 35).

\section{Magnetic resonance imaging}

Twenty-four MRIs conducted within the predefined corrected gestational age of $40-44 \mathrm{wk}$ in each group were assessed $(n=48$; Table 3$)$. MRIs done outside the predefined corrected

Table 2. Brain injury severity for each of the sequential and the overall cUS score as assessed by central reading

\begin{tabular}{|c|c|c|c|c|c|c|c|}
\hline & Day 1 & Day 4 & Day 7 & Day 14 & Day 35 & TEA & Overall cUS score \\
\hline \multicolumn{8}{|l|}{ Experimental group $(n=80)$} \\
\hline No scan/central score $(n)$ & 6 & 7 & 4 & 9 & 4 & 24 & - \\
\hline No injury $(n)$ & 54 & 42 & 44 & 30 & 32 & 10 & 21 \\
\hline Mild/moderate injury $(n)$ & 19 & 22 & 22 & 27 & 29 & 31 & 49 \\
\hline \multicolumn{8}{|l|}{ Control group $(n=77)$} \\
\hline No scan/central score $(n)$ & 6 & 3 & 3 & 4 & 10 & 12 & - \\
\hline Death $(n)$ & 0 & 3 & 9 & 13 & 15 & 19 & - \\
\hline No injury $(n)$ & 55 & 41 & 36 & 26 & 23 & 23 & 26 \\
\hline Mild/moderate injury $(n)$ & 10 & 18 & 18 & 24 & 19 & 17 & 33 \\
\hline $\begin{array}{l}\text { Odds ratio } \\
\text { (95\% confidence interval) }\end{array}$ & $0.15(0.02-1.27)$ & $0.54(0.20-1.46)$ & $0.52(0.19-1.44)$ & $0.70(0.26-1.92)$ & $0.41(0.14-1.22)$ & $0.81(0.23-2.88)$ & $0.47(0.20-1.09)$ \\
\hline
\end{tabular}


Table 3. MRI brain injury severity between experimental and control group

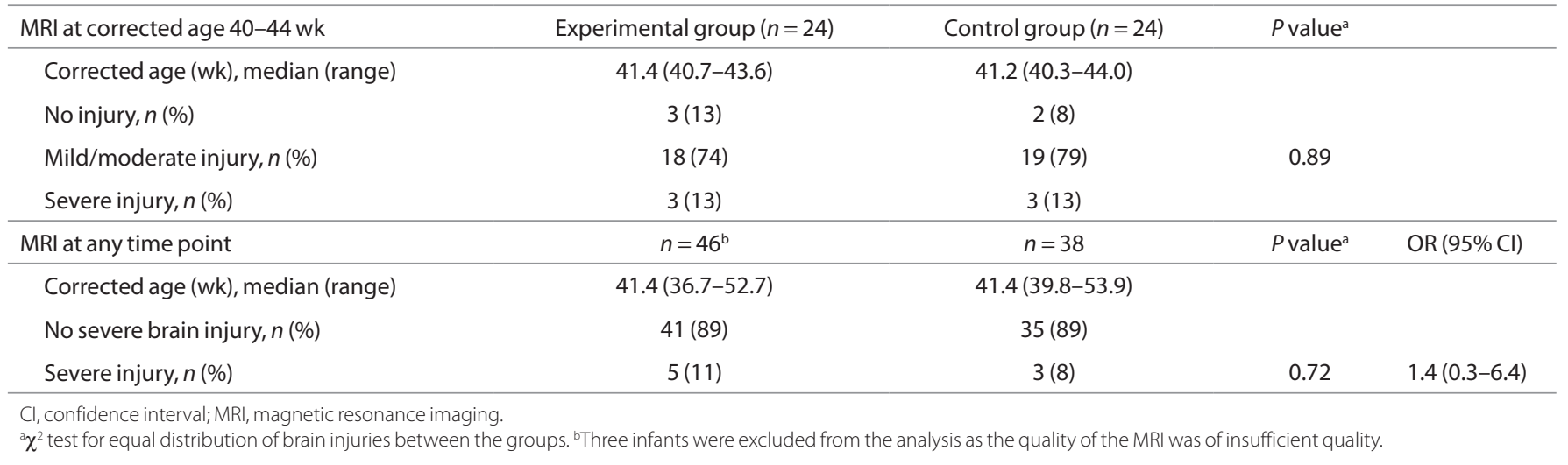

gestational age of $40-44 \mathrm{wk}(n=30$ there of three with artifacts) and MRIs with movement artifacts $(n=4)$ or slice thickness above $2 \mathrm{~mm}$ unless severe injury was clearly seen $(n=5)$ were excluded. MRI brain injury scores did not differ between the two groups. Analysis including MRI at any time point (46 infants in the experimental group and 38 infants in the control group) was also carried out, and there was no difference between the groups (Table 3 ).

\section{Discrepancies between cUS and MRI findings}

None of the cUSs or MRIs scored as no brain injury was scored as severe in the other imaging modality, but in five of the MRI conducted within the predefined gestational age of 40 to $44 \mathrm{wk}$, there was disagreement of mild/moderate in one modality compared to severe brain injury in the other. Two preterm infants with IVH grade 1 or 2 seen on cUS (mild/moderate brain injury) on day 1 and day 7, respectively, were classified as severe brain injury on MRI (Kidokoro score of 12) (16). Both had cerebellar hemorrhages and additionally increased subarachnoid space or cerebral occipital atrophy, which was not identified by cUS.

One preterm infant had IVH grade III (day 7) and subsequently developed posthemorrhagic ventricular dilatation on cUS. MRI at TEA confirmed posthemorrhagic ventricular dilatation; however, according to the MRI Kidokoro scoring system, this resulted in mild/moderate global injury score, since the ventricles had returned to almost normal sizes (16). Cerebellar hemorrhage was seen on cUS in one preterm infant resulting in a severe cUS injury score. As there were no additional lesions and the cerebellar hemorrhage was unilateral on MRI, this resulted in mild/moderate global MRI injury score (16). A lenticulo-striatal stroke was seen on cUS in one preterm infant, this was classified as severe cUS brain injury. On MRI at TEA, the atrophy of the stroke area could be seen; however, in the global brain injury score, the sum of all lesions yielded a mild/moderate injury score.

Twenty-seven MRIs performed at other time points than corrected gestational age 40 to $44 \mathrm{wk}$ were assessed. Four of these had severe brain injury on cUS, which in two of the cases also was identified on MRI. In the other two infants with severe brain injury on cUS, one infant was diagnosed on day 1 with unilateral hemorrhagic infarction; however, the MRI at $49 \mathrm{wk}$ showed mild/moderate injury. The other infant was diagnosed with a lenticulostriatal stroke on cUS day 4 and the MRI at 47 wk of corrected gestational age showed mild/moderate injury.

\section{Feasibility \\ Cranial ultrasound}

Eight-hundred and thirteen of the 887 planned cUS series (cUSs as described in the cUS-standard operating procedure from birth to time of death or TEA) were available for central reading, each series consisting of up to 12 images. In 32 series, the quality was too poor for central image analysis. Seven-hundred and eighty-one (88\%) of the planned cUS series were thus scored centrally. The number of uploaded and centrally scored cUS series was highest on day 7 (experimental group 95\%, control group 96\%) and lowest at TEA (experimental group 66\%, control group 79\%; Figure 1). In total, only $55 \%$ of the infants in the experimental and $65 \%$ in the control group had all cUS from day 1 till TEA or death.

Local overall cUS score was calculated in 165 out of 166 infants; 1 infant died prior to the first cUS. One-hundred and fifty-seven (157/166) infants (95\%) had an overall central cUS score. Reasons for no central overall cUS score were death prior to the first cUS $(n=1)$, too few uploaded image series per patient (less than $3 \mathrm{cUS} ; n=1$ ), poor quality of the images $(n=3)$, or a combination of both $(n=4)$. Three of the infants without an overall central cUS score died before TEA, two in the experimental and one in the control group.

\section{Magnetic resonance imaging}

Eighty-seven (65\%) of the 134 infants alive at TEA had an MRI. Median (range) corrected age at the time of the MRI was $41.4 \mathrm{wk}$ (range: 36.8-53.9). Fifty-seven (66\%) of the MRIs were conducted within the predefined corrected gestational age of 40 to $44 \mathrm{wk}$ (median (range)) 41.4wk (40.0-44.0) in accordance with the MRIstandard operating procedure. The reasons for no MRI were: lack of parental consent $(n=29)$, the infant could not lie still $(n=3)$, or other reasons such as technical problems, child discharged to other department, or failure to turn up at the appointment $(n=15)$.

\section{Central vs. local readings cUS}

Kappa values between the local and central readers were highest for periventricular hemorrhagic infarction $(\kappa=0.79$ at day 7 and 0.75 at day 14) and posthemorrhagic ventricular 


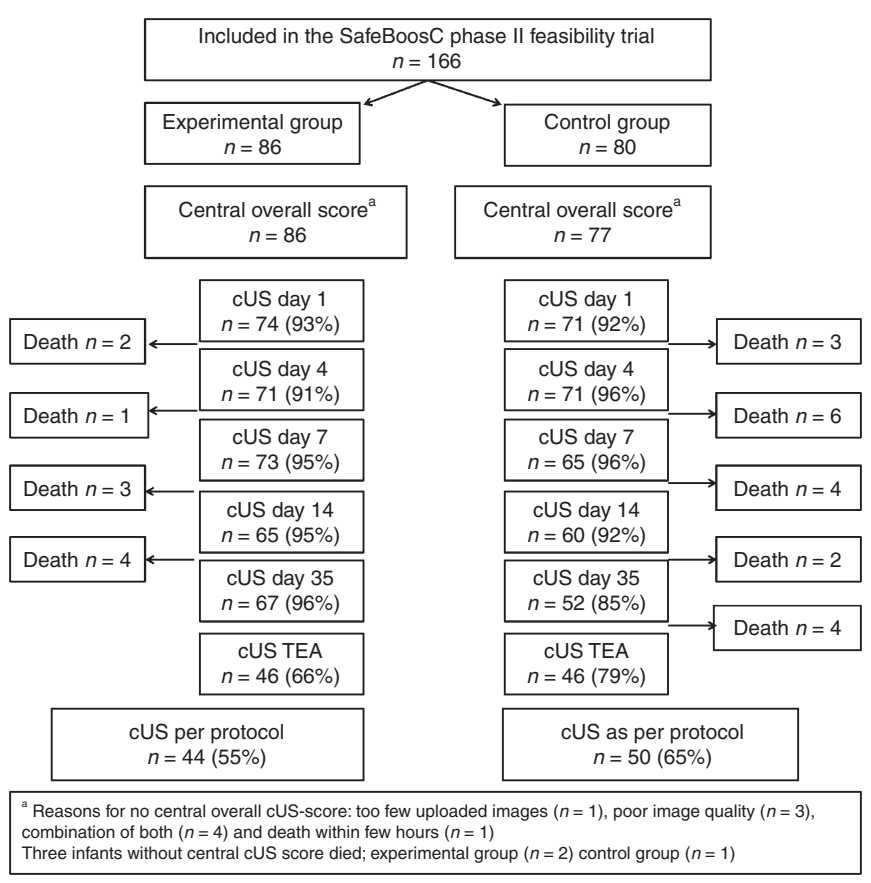

Figure 1. Flow chart from inclusion to term-equivalent age, number of uploaded serial cranial ultrasound scans in the two treatment groups.

dilatation ( $\kappa=1$ at day 7 and 0.72 at day 14 ), whereas the lowest $\kappa$ values were found for intraventricular hemorrhage grade I and II ( $\kappa=0.32$ at day 1 and 0.34 at day 4$)$ and persisting inhomogeneous flaring at day $14(\kappa=0.04)$. Kappa values $(95 \%$ confidence interval) for the overall cUS score no, mild/moderate or severe brain injury was $0.61(0.49-0.72)$ for the central readers and $0.40(0.29-0.51)$ for local vs. central. Interobserver $\kappa$ value for the overall cUS score for severe vs. no severe injury between the two central readers was $0.86(0.75-0.96)$, whereas the agreement between the local and central reading was 0.57 $(0.41-0.74)$. These $\kappa$ values were similar for the images scored in DICOM- and in other formats.

\section{DISCUSSION}

\section{Differences in Brain Injury Between Groups}

The odds ratio of severe brain injury vs. no severe brain injury was insignificant at all time points and for the overall cUS score. In addition, conventional MRI did not suggest any differences between the two groups. However, cUS findings were only a secondary outcome and MRI an explorative outcome in the SafeBoosC II trial; therefore, the study was not powered to look at differences in brain injury between the two groups. More infants in the control group than in the experimental group had severe brain injuries at day 1 , although the patient characteristics were similar. As the infants did not have cUS before inclusion of the trial, it is not possible to determine if these numbers reflect an early effect of the intervention or if it was present before the inclusion. Therefore, inclusions immediately after birth or neuroimaging before intervention are considerations for future studies.

The number of severe brain injury as assessed by blinded central cUS reading was lowest in the experimental group, mainly due to fewer cases of severe intracranial hemorrhages, as compared with the control group during the first week of life. However, there were more mild injuries on cUS in the infants in the experimental group compared with the control group. This could be a result of a shift from severe brain injury and death, which was highest in the control group, toward more mild injuries. This finding was not confirmed on MRI at TEA.

There is no gold standard for monitoring cerebral tissue oxygenation; therefore, the target values of cerebral oxygenation (55-85\%) in the SafeBoosC II trial are based on the best possible evidence, which is the $95 \%$ confidence interval of cerebral NIRS values in 439 preterm infants born below $32 \mathrm{wk}$ of gestation within the first $3 \mathrm{~d}$ of life (P. Lemmers and F. van Bel, unpublished data). In the SafeBoosC II trial, NIRS monitoring in combination with a dedicated treatment guideline for cerebral tissue oxygenation out of range was able to significantly reduce the burden of cerebral hypoxia during the first $72 \mathrm{~h}$ of life (5). It has previously been reported that low cerebral NIRS values in preterm infants during the first days of life are associated with higher grades of intraventricular hemorrhage and lower developmental quotients in very preterm infants $(1,2,17)$, but in this study, we did not find a difference in the number of severe brain injuries between the experimental and the control groups neither in cUS nor in cerebral MRI. However, it must be kept in mind that the SafeBoosC II trial was not powered to look at the numbers of severe brain injuries between groups, as cUS and MRI were secondary and explorative outcomes, it could be speculated that the reduction of the cerebral burden of hypoxia was not of clinical relevance, but the burden was reduced by $58 \%$ in the experimental group (5). The cerebral oxygenation was only measured during the first $72 \mathrm{~h}$ of life, during the period where most severe intracranial hemorrhages are known to occur (7). We did find more severe intracranial hemorrhages in the control group than in the experimental group, but the difference was insignificant. Measuring NIRS during the first $72 \mathrm{~h}$ of life in extremely preterm infants may potentially reduce the number of severe brain injuries especially the early and intracranial hemorrhages, known to cause unfavorable neurodevelopmental outcomes, but the SafeBoosC II trial is not powered to make any final conclusions. Assessment of neurodevelopmental outcome at $2 \mathrm{y}$ of age in this cohort is ongoing and will provide further information on the possible adverse effects of low cerebral oxygenation during the first $72 \mathrm{~h}$ of life in this population.

\section{Feasibility of Neuroimaging in This Multicenter Trial}

We showed that it is feasible though challenging to use central reading of cUS as an outcome measure in a randomized multicenter trial: most images could be centrally scored. Central reading of cUS in multicenter studies has previously proven possible (18). The percentage of uploaded cUS series decreased from day 7 onwards; at TEA, it was only $72 \%$, and many infants were discharged or transferred to local hospitals at this age. As some lesions, such as cystic periventricular leukomalacia (19), cerebral atrophy (20), and posthemorrhagic ventricular dilatation develop over time, it has been argued that it is necessary 
to perform serial cUS until TEA in order to capture the full extent of brain injury (21). However, in one study, only $4 \%$ of the severe brain injuries was detected after day 35 (19). None of our infants scanned at TEA shifted category from no severe brain injury to severe brain injury, but we may potentially have missed some cases.

MRI at TEA was optional and performed in only $64 \%$ of the infants. This number was lower than we had expected, maybe because MRI at TEA was not standard clinical care of preterm infants in most participating centers, and an additional informed consent for MRI was required separately from the consent to the randomized intervention. This is a limitation of our study. The infants in the SafeBoosC II trial were followed closely by serial cUS, and it is possible that the parents felt they were already well informed about the extent of the brain injury and the risk of later adverse neurodevelopmental outcomes and therefore did not want to take part in an additional MRI scan where the infant might be sedated and admitted to the hospital for this purpose alone. In this study, two infants who had mild/moderate brain injury on early cUS because of IVH grade I-II findings were categorized as having severe injuries on MRI because of cerebellar hemorrhages that were not recognized on serial cUS. Therefore, MRI is important in addition to cUS (21). Furthermore, MRI at TEA is superior to cUS in predicting the risk of CP in preterm infants (10) and contributes with additional information to serial cUS when estimating risk of adverse neurodevelopmental outcome (18) - MRI at TEA therefore plays an important role in the risk stratification in these infants (11). In our study, the number of severe and mild/moderate brain injuries on MRI was similar in the control and the experimental groups.

In future, large multicenter randomized controlled trials studying neuroprotective interventions; only MRI-dedicated neuroimaging groups/centers might use MRI. The MRI protocol should be followed rigorously, in order to make sure that the timing, slice thickness, and quality are comparable for all infants. With high resolution MRI, mild injury might be easier to pick up and fewer patients are needed to prove differences in quantitative measures that are related to long-term outcome (22). It is essential to obtain knowledge on the possible effects (benefits or harms) of the neuroprotective interventions in the vulnerable and developing preterm brain. In this study, it was not possible to perform quantitative MRI analysis because of different scanner systems and image acquisitions.

\section{Central vs. Local Readings cUS}

The overall $\kappa$ values for the local and the central readers were moderate to good for severe injuries and poor to moderate for $\mathrm{mild} /$ moderate injuries. These findings are consistent with previously published interobserver cUS studies (23-25).

Local readers have the advantage of real-time images, whereas the central readers have only selected still images for assessment. However, central readers are blinded to the treatment groups, which are important in randomized clinical trials where blinding of the person performing the cUS is impossible, as in the SafeBoosC II trial. Central reading might be thus the preferred method of analyzing cUS images in randomized multicenter studies (24) without blinded intervention. The problems of poor image quality may be addressed by dedicated training in a future study.

\section{Conclusions}

The distribution of brain injury into no, mild/moderate, or severe injury did not differ among the groups neither for the overall cUS score nor for MRI. There was less severe brain injury in the experimental group as assessed by central reading of cerebral ultrasound, which is in line with the reduction of cerebral hypoxia in the experimental group of the SafeBoosC II trial, but MRI conducted at TEA in 64\% of the infants did not support this finding. The problems in acquiring cUS and MRI data of good quality might be addressed by dedicated training. cUS as well as MRI images at TEA were often missing. Central, blinded readers of cUS achieved high interobserver agreement for severe brain injury. Large randomized clinical trials should be performed to evaluate the effect of the burden of cerebral hypoxia on brain injury in extremely preterm infants.

\section{METHODS}

\section{Study Design and Population}

One-hundred and sixty-six extremely preterm infants from eight European countries were included (1 June 2012 to 31 December 2013) (5). The infants were randomized to either the experimental group or the control group. Infants in the experimental group were monitored with NIRS, and continuous cerebral tissue oxygenation levels were available for the clinician together with a dedicated treatment guideline (4), listing possible interventions if the cerebral oxygenation level was out of range (55-85\%). The infants in the control group were also monitored with cerebral NIRS, and the data were recorded but blinded to the clinicians. They were given standard care. In both groups, the cerebral NIRS monitoring was started within $3 \mathrm{~h}$ after birth and continued until $72 \mathrm{~h}$ of life (5).

The protocol of SafeBoosC II is published (3) and is available in full at www.safeboosc.eu. The study is registered at ClinicalTrial.gov, NCT01590316.

\section{Cranial Ultrasound}

On day 1 (any time during the first $24 \mathrm{~h}$ of life), $4( \pm 1), 7( \pm 1), 14( \pm 1)$, $35( \pm 1$ ), and at TEA (week 38 to 44 ) standardized cUS (six coronal and five sagittal images through the anterior fontanel and 1 through the mastoid window) was performed. The cUS was performed unblinded and according to local standard, either by the clinical staff or by a suitable qualified sonographer. Local unblinded investigators reported the cUS findings in an electronic record form. The images were anonymized and uploaded as DICOM files for central reading. The local scorings from the electronic record form were converted into predefined diagnoses (Table 4), which correlate to later developmental outcomes $(18,20,26,27)$.

Central reading was performed by two cUS readers (M.B. and C.H.) blinded to treatment group and medical history of the infants. Initial cUS images from two times 20 random infants were scored by the central readers to ensure common use of the scoring system. Then, the central readers scored all cUS series individually according to the predefined diagnoses (Table 4). Disagreements were resolved by consensus meetings. Each cUS was categorized on a three-level scale as no brain injury, mild/moderate brain injury, or severe brain injury $(18,20,26,27)$. DICOM images were analyzed using the software program OsiriX version 6.0. (Pixmeo, Geneva, Switzerland). Images uploaded in other formats were viewed in Preview.

The scores of all cUS images for each infant were combined into an overall cUS score reflecting the worst scan in any of the serial cUSs. In case of death, the overall cUS score would reflect the worst scan in 
Table 4. Predefined diagnosis for cranial ultrasound scans

\begin{tabular}{l} 
No brain injury \\
- None of the findings below \\
Mild/moderate brain injury \\
- Grade 1-2 IVH (including germinal layer hemorrhage) \\
- Isolated ventriculomegaly with ventricular index $<\mathrm{p} 97^{\mathrm{a}}$ \\
- Inhomogeneous flaring persisting after day 7 \\
- Global thinning of corpus callosum at TEA \\
Severe brain injury \\
- IVH grade III (ventricular index $>\mathrm{p} 97$ during the acute phase) \\
- Posthemorrhagic ventricular dilatation \\
- Parenchymal/periventricular hemorrhagic infarction \\
- Unilateral porencephalic cysts \\
- Cystic periventricular leukomalacia (bilateral) \\
- Cerebellar hemorrhage \\
- Cerebral atrophy at term age ${ }^{\mathrm{b}}$ \\
- Stroke \\
\hline At each cUS the infant was diagnosed with one of the above findings. \\
CUS, cranial ultrasound; IVH, intraventricular hemorrhage;TEA, term-equivalent age. \\
oReference values according to Brouwer et al. (21). bBrain atrophy was defined as the \\
combination of enlarged subarachnoid spaces, widened interhemispheric fissure, and \\
reduction in complex gyral folding with or without concomitant ventriculomegaly, \\
according to Horsch et al. (20).
\end{tabular}

any of the cUSs performed before the time of death. Infants missing cUS examinations on more than two of the predefined time points were excluded from the analysis unless the brain injury was classified as severe in any of the existing images.

\section{Magnetic Resonance Imaging}

At TEA (corrected age 40-44 wk), the parents of the preterm infants were offered an MRI. The MRI procedure was performed according to local protocols; preferably on a 3T MRI system; if this was not available, images were acquired on a $1.5 \mathrm{~T}$ system. $\mathrm{T}_{1}$ - and $\mathrm{T}_{2}$-weighted images were used for brain injury scoring. The MRIs were anonymized and uploaded in DICOM format for central reading. A.M.P., C.H., M.F., and M.B. analyzed the MRIs blinded to treatment group, medical history of the infant, and cUS findings. MRIs were scored in consensus. The scoring was done according to a combination of previously published scoring systems $(11,16,28)$. The scores were categorized into: normal brain ( $0-3$ points), mild/moderate brain injury (4-11 points), and severe brain injury (12 points or more) as described by Kidokoro (16). All MRIs were evaluated in the software program OsiriX version 6.0.

\section{Ethics}

The SafeBoosC phase II feasibility multicenter trial was approved by each hospital's local research ethics committee (Hopital Femme Mere Enfants, Lyon, France; Rigshospitalet, Copenhagen, Denmark; La Paz University Hospital, Madrid, Spain; Cork University Maternity Hospital, Cork, Ireland; Wilhelmina Children's Hospital, Utrecht, The Netherlands; Medical University of Graz, Graz, Austria; Fondazione IRCCS Ca' Granda Ospedale Maggiore Policlinico, Milan, Italy; and Rosie Hospital, Cambridge University Hospitals, UK) and where required (Austria, Denmark, and France) by the competent authority responsible for medical devices. Parental written informed consent was mandatory before inclusion in the trial. Additional informed parental consent was obtained for MRI at TEA if MRI was not clinically indicated or standard clinical care.

\section{Statistics}

Patient characteristics were summarized. The distribution of the brain injury severity on each day of the sequential cUS scans, the overall
cUS score, and the MRI scores was tested between the groups by $\chi^{2}$ test. The results were dichotomized into severe brain injury or no severe brain injury, and odds ratios were calculated. The percentage of the per-protocol images that were uploaded and scored was calculated for each day of the sequential cUS and for MRI. Kappa statistics was used to test the agreement between the two central readers and between the central and the local cUS readers. The statistics was performed using the software RStudio Version 0.98.501 (Boston, MA) and IBM SPSS Statistics for Windows Version 20.0.0 (Armonk, NY).

\section{STATEMENT OF FINANCIAL SUPPORT}

The Danish Council for Strategic Research financially supported this work through an unconditional and unrestricted grant of DKK 11,100,105. The funder had no role in the design, conduct, or analysis of the trial.

Disclosure: The authors have no financial relationships relevant to this article to disclose.

\section{REFERENCES}

1. Noori S, McCoy M, Anderson MP, Ramji F, Seri I. Changes in cardiac function and cerebral blood flow in relation to peri/intraventricular hemorrhage in extremely preterm infants. J Pediatr 2014;164:264-70.e1-3.

2. Alderliesten T, Lemmers PM, van Haastert IC, et al. Hypotension in preterm neonates: low blood pressure alone does not affect neurodevelopmental outcome. J Pediatr 2014;164:986-91.

3. Hyttel-Sorensen S, Austin T, van Bel F, et al. A phase II randomized clinical trial on cerebral near-infrared spectroscopy plus a treatment guideline versus treatment as usual for extremely preterm infants during the first three days of life (SafeBoosC): study protocol for a randomized controlled trial. Trials 2013;14:120.

4. Pellicer A, Greisen G, Benders M, et al. The SafeBoosC phase II randomised clinical trial: a treatment guideline for targeted near-infrared-derived cerebral tissue oxygenation versus standard treatment in extremely preterm infants. Neonatology 2013;104:171-8.

5. Hyttel-Sorensen S, Pellicer A, Alderliesten T, et al. Cerebral near infrared spectroscopy oximetry in extremely preterm infants: phase II randomised clinical trial. BMJ 2015;350:g7635.

6. de Vries LS, van Haastert IC, Benders MJ, Groenendaal F. Myth: cerebral palsy cannot be predicted by neonatal brain imaging. Semin Fetal Neonatal Med 2011;16:279-87.

7. Bassan $\mathrm{H}$, Benson $\mathrm{CB}$, Limperopoulos $\mathrm{C}$, et al. Ultrasonographic features and severity scoring of periventricular hemorrhagic infarction in relation to risk factors and outcome. Pediatrics 2006;117:2111-8.

8. Munck P, Haataja L, Maunu J, et al.; PIPARI Study Group. Cognitive outcome at 2 years of age in Finnish infants with very low birth weight born between 2001 and 2006. Acta Paediatr 2010;99:359-66.

9. de Vries LS, Benders MJ, Groenendaal F. Imaging the premature brain: ultrasound or MRI? Neuroradiology 2013;55:Suppl 2:13-22.

10. Mirmiran M, Barnes PD, Keller K, et al. Neonatal brain magnetic resonance imaging before discharge is better than serial cranial ultrasound in predicting cerebral palsy in very low birth weight preterm infants. Pediatrics 2004;114:992-8.

11. Woodward LJ, Anderson PJ, Austin NC, Howard K, Inder TE. Neonatal MRI to predict neurodevelopmental outcomes in preterm infants. N Engl J Med 2006;355:685-94.

12. Rutherford M, Ramenghi LA, Edwards AD, et al. Assessment of brain tissue injury after moderate hypothermia in neonates with hypoxic-ischaemic encephalopathy: a nested substudy of a randomised controlled trial. Lancet Neurol 2010;9:39-45.

13. Ment LR, Hirtz D, Hüppi PS. Imaging biomarkers of outcome in the developing preterm brain. Lancet Neurol 2009;8:1042-55.

14. Pandit AS, Ball G, Edwards AD, Counsell SJ. Diffusion magnetic resonance imaging in preterm brain injury. Neuroradiology 2013;55:Suppl 2:65-95.

15. Anjari M, Srinivasan L, Allsop JM, et al. Diffusion tensor imaging with tract-based spatial statistics reveals local white matter abnormalities in preterm infants. Neuroimage 2007;35:1021-7.

16. Kidokoro H, Neil JJ, Inder TE. New MR imaging assessment tool to define brain abnormalities in very preterm infants at term. AJNR Am J Neuroradiol 2013;34:2208-14. 


\section{Articles | Plongaard et al.}

17. Sorensen LC, Maroun LL, Borch K, Lou HC, Greisen G. Neonatal cerebral oxygenation is not linked to foetal vasculitis and predicts intraventricular haemorrhage in preterm infants. Acta Paediatr 2008;97:1529-34.

18. Hintz SR, Barnes PD, Bulas D, et al.; SUPPORT Study Group of the Eunice Kennedy Shriver National Institute of Child Health and Human Development Neonatal Research Network. Neuroimaging and neurodevelopmental outcome in extremely preterm infants. Pediatrics 2015;135:e32-42.

19. De Vries LS, Van Haastert IL, Rademaker KJ, Koopman C, Groenendaal F. Ultrasound abnormalities preceding cerebral palsy in high-risk preterm infants. J Pediatr 2004;144:815-20.

20. Horsch S, Muentjes C, Franz A, Roll C. Ultrasound diagnosis of brain atrophy is related to neurodevelopmental outcome in preterm infants. Acta Paediatr 2005;94:1815-21.

21. Brouwer MJ, van Kooij BJ, van Haastert IC, et al. Sequential cranial ultrasound and cerebellar diffusion weighted imaging contribute to the early prognosis of neurodevelopmental outcome in preterm infants. PLoS One 2014;9:e109556.

22. Ball G, Boardman JP, Arichi T, et al. Testing the sensitivity of tract-based spatial statistics to simulated treatment effects in preterm neonates. PLoS One 2013;8:e67706.

23. Hagmann CF, Halbherr M, Koller B, Wintermark P, Huisman T, Bucher HU; Swiss Neonatal Network. Interobserver variability in assessment of cranial ultrasound in very preterm infants. J Neuroradiol 2011;38:291-7.

24. Hintz SR, Slovis T, Bulas D, et al.; NICHD Neonatal Research Network. Interobserver reliability and accuracy of cranial ultrasound scanning interpretation in premature infants. J Pediatr 2007;150:592-6, 596. e1-5.

25. Kuban K, Adler I, Allred EN, et al. Observer variability assessing US scans of the preterm brain: the ELGAN study. Pediatr Radiol 2007;37:1201-8.

26. Benders MJ, Groenendaal F, De Vries LS. Preterm arterial ischemic stroke. Semin Fetal Neonatal Med 2009;14:272-7.

27. Bolisetty S, Dhawan A, Abdel-Latif M, Bajuk B, Stack J, Lui K; New South Wales and Australian Capital Territory Neonatal Intensive Care Units' Data Collection. Intraventricular hemorrhage and neurodevelopmental outcomes in extreme preterm infants. Pediatrics 2014;133:55-62.

28. Childs AM, Ramenghi LA, Cornette L, et al. Cerebral maturation in premature infants: quantitative assessment using MR imaging. AJNR Am J Neuroradiol 2001;22:1577-82.

(c) (i) () () This work is licensed under a Creative Commons cc) Attribution-NonCommercial-NoDerivs $\quad 4.0$ International License. The images or other third party material in this article are included in the article's Creative Commons license, unless indicated otherwise in the credit line; if the material is not included under the Creative Commons license, users will need to obtain permission from the license holder to reproduce the material. To view a copy of this license, visit http://creativecommons.org/licenses/by-nc-nd/4.0/ 